\title{
Effect of Fluctuating Surface Temperature on Natural Convection Flow Over Cylinders of Elliptic Cross Section
}

\author{
M. K. Jaman ${ }^{1}$ and M. A. Hossain ${ }^{2, *}$ \\ ${ }^{I}$ Department of Mathematics, University of Dhaka, Dhaka-1000, Bangladesh \\ ${ }^{2}$ Department of Mathematics, COMSATS Institute of Information Technology, Islamabad, Pakistan
}

\begin{abstract}
Effect of small amplitude oscillation in the wall temperature on the natural convection flow from a cylinder of elliptic cross section has been investigated. Solutions of the governing equation are obtained for eccentric angle $\alpha$ in the range $[0, \pi]$ employing the finite difference method together with Keller-box elimination. The solutions are discussed in terms of amplitude and phase of the skin-friction and rate of heat transfer for fluid having Prandtl number equals 0.1 for given values of different $A_{0}$ that represent the ratio of the semi-major and semi-minor axis of the cylinder.
\end{abstract}

Keywords: Natural convection flow, elliptic cylinders and fluctuating flow.

\section{INTRODUCTION}

Free convection refers to a case where the fluid movement is created by the warm fluid itself and also a process in which the fluid motion is set up by buoyancy effects due to density gradients within the fluid caused by the temperature field itself. The density of fluid decreases as it is heated; thus, hot fluids are lighter than cool fluids. Warm fluid surrounding a hot objects rises, and is replaced by cooler fluid. The result is a circulation of a fluid above the warm surface. The requirements of modern technology have stimulated interest in fluid flows, which involve the interaction of applicable phenomena. For example, free convection over vertical or horizontally placed cylinders is relevant to flow over tubes in nuclear reactors and cylindrical heating elements. With this understanding, Merkin [1] considered the case of free convection boundary layer on an isothermal horizontal circular cylinder in viscous fluid and was the first to present a complete solution to this problem for Newtonian fluid using Blasius and Görtler series expansion methods along with an integral method and a finite difference scheme. The case of free convective boundary layers on cylinder of elliptic cross section over isothermal bodies had been considered, later, by Merkin [2]. In this investigation the heat transfers from isothermal cylinder of elliptic cross section of various eccentricities in both cases when the major axis is horizontal and vertical had been considered. Hossain et al. [3] investigated the same problem posed above for steady free convection flow by bringing the effect of radiative heat transfer. In that work, they showed that the rate of heat transfer from the slender body is higher than from the blunt body due to increase of radiation parameter. Saville and Churcill [4] and Lin and Chao [5] had also investigated free convection flow

*Address correspondence to this author at the Department of Mathematics, University of Dhaka, Dhaka-1000, Bangladesh; Tel: +8802-8315840;

E-mail: anwar@univdhaka.edu from a horizontal cylinder and from axisymmetric bodies of arbitrary contours with isothermal surface condition.

In unsteady boundary layer theory, one area of study, has received much attention in the past deals with boundary layers responses to imposed oscillations. Lighthill [6] was the first to have studied the unsteady forced flow of a viscous incompressible fluid past a flat plate and a circular cylinder with small amplitude oscillation in the free stream. Also Glaurt and Lighthill [7] investigated the axisymmetric boundary layer flow past a long thin circular cylinder. Subsequent authors have extended Lighthill's solution, but in general have retained the small- $\varepsilon$ approximations $(0 \leq \varepsilon \leq 1$ is the amplitude parameter). Gibellato [8] and Ghosh [9] restricted themselves to the case of the semi-infinite plate, and independently extended for small- $\xi\left(=\omega x / \underline{U}_{0}(x)\right.$, the frequency parameter) expansion to several terms. Ghosh also extended the large- $\xi$ expansion, but considered only the viscous not the thermal boundary layer. Verma [10] investigated the fluctuating free convection fluctuating boundary layer on a horizontal plate subject to small amplitude oscillation about a constant mean in the surface temperature. These oscillatory flow and heat transfer problems are important in engineering because such flows occur often in practice. Similar analysis, using the Karman-Paulhausen approximate integral method, effect of surface temperature oscillation on the oscillating natural convection flow from a vertical surface had been made by Nanda and Sharma [11]. They consider skin friction and the rate of heat transfer for both low and high frequency. Harpole and Catton [12] considered the steady laminar natural convection flow over a heated blunt body for low Prandtl number. They applied the Blasius series expansion method to convert the partial differential equation into ordinary differential equation. The results were present for both the suitable surface heat flux and the suitable surface temperature case. Based on the linearized theory, Kelleher and Yang [13] have studied the heat transfer responses of a laminar free convection boundary layer along 
a vertical heated plate to surface temperature oscillations, when the mean surface temperature $\theta_{w}(x)$ is proportional to $x^{n}$, where $x$ is the distance measured from the leading edge of the plate. The results were presented in terms of skin friction and heat transfer for small $\varepsilon$, which measures the distribution of the frequency of oscillation in the surface temperature and buoyancy force. Later, Eshghy et al. [14] investigated the finite amplitude longitudinal oscillation on free convection flow of a viscous incompressible fluid from a vertical surface. Hossain et al. [15] investigate the buoyancy force arising from both thermal and mass diffusion in the unsteady natural convection flow from a vertical plate based on the linearization theory posed by Kelleher and Yang [13]. The surface is subjected to small amplitude temporal oscillations in both its temperature and species concentration with nonzero means. In the study they employed the implicit finite difference method to obtaining the solution for intermediate frequencies. Numerical results are obtained for a wide range of the frequency of oscillation. The problem posed in [13] has further been investigated by Hossain et al. [16] for the electrically conducting fluid along a vertical plate in the presence of the variable transverse magnetic field assuming that the mean temperature assumed to vary as power of $x$ that measure the distance from the leading edge. Very recently Jaman et al. [17] dealt with the problems encountered in the flow field at the free convection flow of a viscous incompressible fluid along an infinite horizontal cylinder when the temperature of the cylinder is oscillating sinusoidically. In which they investigated the local skin friction and local rate of heat transfer in terms of amplitude and phase, respectively. The flow patterns had also been shown graphically in terms of skin-friction and heat transfer coefficient with effect of Prandtl number, Pr, the Strouhal number, St, amplitude of oscillation, $\varepsilon$, and curvature parameter $\xi$.

In the present paper, we investigate the natural convection boundary layer flow of a viscous incompressible fluid over an isothermal cylinder of elliptic cross section of various eccentricities in both cases when the major axis is horizontal as well as vertical. The temperature of the cylinder is assumed to be oscillating about a mean temperature $G_{0}$, with small amplitude, $\varepsilon$. The motion is caused by the action of oscillating buoyant body forces on the fluid near the cylinder. Using appropriate transformations, the governing equations reduced to local non-similarity equations for both the steady mean flow and for the oscillating flow, solutions of which are obtained numerically employing the finite difference method together with Keller-box elimination method.
Here the heat transfer results for a range of values of the pertinent parameters have been represented. Effects of pertinent parameters, such as the frequency parameter, $\omega$, and for the unit value of the Prandtl number, $\mathrm{Pr}$, on the local skinfriction as well as on the local heat transfer coefficients, obtained for steady state situation, are shown graphically for the entire surface of the cylinder. The results are presented in terms of amplitude and phase in surface heat-flux coefficient temperature for the values of $\alpha$ lies in $[0, \pi]$ radian which measure the eccentric angle. The flow patterns in terms of streamlines and isotherms has also been shown graphically with effect of Prandtl number $\operatorname{Pr}$, the frequency of oscillation, $\omega$, amplitude of oscillation, $\varepsilon$, and the different values of $A_{0}$.

\section{MATHEMATICAL FORMULATION}

We consider a two-dimensional unsteady laminar free convection boundary layer flow of a viscous incompressible fluid along a cylinder of elliptic cross section with $a$ and $b$ as the semi-major and semi-minor axis of the cylinder respectively. It is assumed that the surface temperature of the cylinder is oscillating with small amplitude about a constant mean temperature. The mean surface temperature of the cylinder is maintained at $\Delta T$ and the ambient temperature of the fluid assumed to be $T_{\infty}$. The physical configuration and the coordinate system for this flow are shown in Fig. (1).

Under the usual Boussinesq approximation the flow is governed by the following boundary layer equations:

$$
\begin{aligned}
& \frac{\partial u}{\partial x}+\frac{\partial v}{\partial y}=0 \\
& \frac{\partial u}{\partial t}+u \frac{\partial u}{\partial x}+v \frac{\partial u}{\partial y}=g \beta \sin \phi\left(T-T_{\infty}\right)+v \frac{\partial^{2} u}{\partial y^{2}} \\
& \frac{\partial T}{\partial t}+u \frac{\partial T}{\partial x}+v \frac{\partial T}{\partial y}=\frac{\kappa}{\rho C_{p}} \frac{\partial^{2} T}{\partial y^{2}}
\end{aligned}
$$

where, $(u, v)$ are the velocity components along the $(x, y)$ axes, $v$ is the kinematic viscosity, $\phi$ the angle made by the outward normal from the cylinder, $T$ being the temperature of the fluid in the boundary layer, $g$ is the gravitational acceleration, $\rho$ is the density of the fluid, $\beta$ is the coefficient of thermal expansion. It is worth mentioning that Bousinesqu approximation is valid for small temperature difference be-
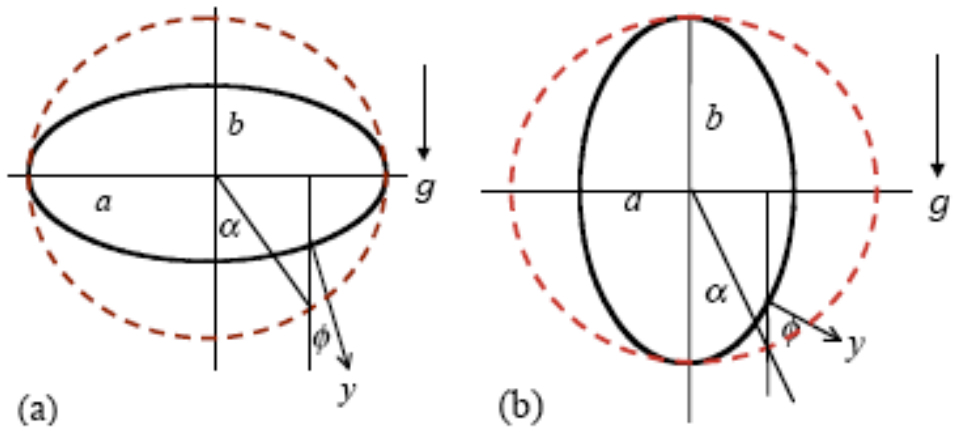

Fig. (1). Physical model and coordinate system of (a) blunt and (b) slender elliptic cylinder. 
tween the surface of the body and the ambient fluid. Hence the present investigation is valid for week buoyancy or smaller values of Grashof number.

The boundary conditions are to be satisfied by the equations (1) to (3) are

$u=0, v=0, \quad T-T_{\infty}=\Delta T F(t)$ at $\mathrm{y}=0$

$u \rightarrow 0, T \rightarrow 0$ as $\mathrm{y} \rightarrow \infty$

In Equation (4) $F(t)$ is an oscillating function in $t$

We now introduce the following non-dimensional quantities

$$
\begin{gathered}
u=\frac{v}{a} G r_{L}^{1 / 2} U, \quad v=\frac{v}{a} G r_{L}^{1 / 4} V, \quad \frac{T-T_{\infty}}{\Delta T}=G \\
Y=v G r_{L}^{1 / 4} y, \quad X=\frac{x}{a}, \tau=\frac{a^{2}}{v} G r^{-1} t \\
G r_{L}=\frac{g \beta \Delta T}{v^{2}} a^{3}
\end{gathered}
$$

The equations then become

$\frac{\partial U}{\partial X}+\frac{\partial V}{\partial Y}=0$

$\frac{\partial U}{\partial \tau}+U \frac{\partial U}{\partial X}+V \frac{\partial U}{\partial Y}=\sin \phi G+\frac{\partial^{2} U}{\partial Y^{2}}$

$\frac{\partial G}{\partial \tau}+U \frac{\partial G}{\partial X}+V \frac{\partial G}{\partial Y}=\frac{1}{\operatorname{Pr}} \frac{\partial^{2} G}{\partial Y^{2}}$

The boundary conditions are then

$U=0, V=0, \quad G=F(\tau)=1+\varepsilon \cos \omega \tau$ at $Y=0$

$U \rightarrow 0, \quad G \rightarrow 0 \quad$ as $\quad Y \rightarrow \infty$

In Equation (9) $\omega$ is the frequency of oscillation, $\varepsilon(<1)$ is a positive real number that designates the amplitude of oscillation in surface temperature and $\tau$ is the time.

To deal with bodied with rounded lower ends for which $\sin \phi / X \rightarrow \mathrm{A}_{0}$ (a constant) as $X \rightarrow 0$. For circular cylindrical cross section $\sin \phi=\sin X$, we treat the surface as an elliptic cylinder of two orientations; namely: (i) a blunt elliptic cylinder and (ii) a slender elliptic cylinder. When the major axis is horizontal then the surface is blunt elliptic cylinder and for slender elliptic cylinder the major axis is vertical. $X$ and $\sin \phi$ are given in terms of a parameter $\alpha$, as shown in Fig. (1), by

$X=\int_{0}^{\alpha} \sqrt{\left(1-e^{2} \sin ^{2} \lambda\right)} d \lambda$ and $\sin \varphi=\frac{A_{0} \sin \alpha}{\left(1-e^{2} \sin ^{2} \alpha\right)^{1 / 2}}$

for a blunt elliptic cylinder and

$$
\begin{aligned}
& X=\int_{0}^{\alpha} \sqrt{\left(1-e^{2} \cos ^{2} \lambda\right)} d \lambda \\
& \text { and } \sin \varphi=\frac{\sin \alpha}{\left(1-e^{2} \cos ^{2} \alpha\right)^{1 / 2}}
\end{aligned}
$$

for a slender elliptic cylinder. For both the cases ' $a$ ' is the length of the semi-major axis, ' $b$ ' is that of the semi-minor axis and $\alpha$ is the eccentric angle with eccentricity, $e=\sqrt{1-(b / a)^{2}}$, so that $A_{0}=b / a$ for the blunt orientation and $A_{0}=(a / b)^{2}$ for slender orientation.

The solution of the above system of differential equations will be obtained in terms of complex functions only, the real parts of which will have physical significance. We write $U$, $V$, and $G$ as the sum of the steady and small oscillating components as

The surface temperature conditions in (9) suggest the solutions of equations (6)-(8) of the form

$$
\begin{aligned}
& U(X, Y)=U_{0}(X, Y)+\varepsilon e^{i \omega \tau} U_{1}(X, Y) \\
& V(X, Y)=V_{0}(X, Y)+\varepsilon e^{i \omega \tau} V_{1}(X, Y) \\
& G(X, Y)=G_{0}(X, Y)+\varepsilon e^{i \omega \tau} G_{1}(X, Y)
\end{aligned}
$$

where $U_{0}, V_{0}$ and $G_{0}$ represent the steady mean flow satisfying the equations:

$$
\begin{aligned}
& \frac{\partial U_{0}}{\partial X}+\frac{\partial V_{0}}{\partial Y}=0 \\
& U_{0} \frac{\partial U_{0}}{\partial X}+V_{0} \frac{\partial U_{0}}{\partial Y}=\sin \varphi G_{0}+\frac{\partial^{2} U_{0}}{\partial Y^{2}} \\
& U_{0} \frac{\partial G_{0}}{\partial X}+V_{0} \frac{\partial G_{0}}{\partial Y}=\frac{1}{\operatorname{Pr}} \frac{\partial^{2} G_{0}}{\partial Y^{2}}
\end{aligned}
$$

with boundary conditions

$$
\begin{aligned}
& U_{0}=V_{0}=0, G_{0}=1 \text { at } Y=0 \\
& U_{0} \rightarrow 0, G_{0} \rightarrow 0 \text { as } y \rightarrow \infty
\end{aligned}
$$

and for the unsteady flow, the components $U_{1}, V_{1}$ and $G_{1}$ satisfy the equations

$$
\begin{aligned}
& \frac{\partial U_{1}}{\partial X}+\frac{\partial V_{1}}{\partial Y}=0 \\
& U_{0} \frac{\partial U_{1}}{\partial X}+U_{1} \frac{\partial U_{0}}{\partial X}+V_{0} \frac{\partial U_{1}}{\partial Y}+V_{1} \frac{\partial U_{0}}{\partial Y}+i \omega U_{1}= \\
& \sin \varphi G_{1}+\frac{\partial^{2} U_{1}}{\partial Y^{2}} \\
& U_{0} \frac{\partial G_{1}}{\partial X}+U_{1} \frac{\partial G_{0}}{\partial X}+V_{0} \frac{\partial G_{1}}{\partial Y}+V_{1} \frac{\partial G_{0}}{\partial Y}+i \omega G_{1}= \\
& \frac{1}{\operatorname{Pr}} \frac{\partial^{2} G_{1}}{\partial Y^{2}}
\end{aligned}
$$

Following are the boundary conditions to be satisfied by the above equations

$$
\begin{aligned}
& U_{1}=V_{1}=0, G_{1}=1 \text { at } Y=0 \\
& U_{1} \rightarrow 0, G_{1} \rightarrow 0 \text { as } Y \rightarrow \infty
\end{aligned}
$$


It should be mention that our desired solutions are the real parts of the function.

To get the set of equations in convenient form for integration, we define the following one parameter group of transformation for the dependent and the independent variables:

$\zeta=X, \quad \eta=Y, \quad\left(\psi_{0}, \psi_{1}\right)=\zeta\left[f_{0}(\zeta, \eta), f(\zeta, \eta)\right]$

$\left(G_{0}, G_{1}\right)=\left[\theta_{0}(\zeta, \eta), \theta(\xi, \eta)\right]$

Where $\psi_{0}$ and $\psi_{1}$ are, respectively, the stream functions for the steady state flow and the oscillating part of the problem defined by

$$
\left(U_{0}, U_{1}\right)=\left(\frac{\partial \psi_{0}}{\partial \eta}, \frac{\partial \psi_{1}}{\partial \eta}\right) \quad\left(V_{0}, V_{1}\right)=\left(-\frac{\partial \psi_{0}}{\partial \zeta},-\frac{\partial \psi_{1}}{\partial \zeta}\right)
$$

that satisfy equations (13) and (17) automatically.

Thus for the steady state flow, the set of equations (14)(15) are transformed to

$$
\begin{aligned}
& f_{0}^{\prime \prime \prime}+f_{0} f_{0}^{\prime \prime}-f_{0}^{\prime 2}+\frac{\sin \phi}{\zeta} \theta_{0}=\zeta\left(f_{0}^{\prime} \frac{\partial f_{0}^{\prime}}{\partial \zeta}-f_{0}^{\prime \prime} \frac{\partial f_{0}}{\partial \zeta}\right) \\
& \frac{1}{\operatorname{Pr}} \theta_{0}^{\prime \prime}+f_{0} \theta_{0}^{\prime}=\zeta\left(f_{0}^{\prime} \frac{\partial \theta_{0}}{\partial \zeta}-\theta_{0}^{\prime} \frac{\partial f_{0}}{\partial \zeta}\right)
\end{aligned}
$$

Here, $\mathrm{Pr}$ is defined to be the ratio of the kinematic viscosity to the thermal diffusivity of the fluid and known as Prandtl number.

The appropriate boundary conditions to be satisfied by (22) and (23) are

$$
\begin{aligned}
& f_{0}(\zeta, 0)=f_{0}^{\prime}(\zeta, 0)=0, \theta_{0}(\zeta, 0)=1 \\
& f_{0}^{\prime}(\zeta, \infty)=0, \quad \theta_{0}(\zeta, \infty)=0
\end{aligned}
$$

Here prime denotes the differentiation with respect to $\eta$.

Now, we are in a position to get the fluctuating parts of the momentum and the energy equations from (18) and (19) using the transformations given in (21). The equations obtained are as given below:

$$
\begin{aligned}
& f^{\prime \prime \prime}+f_{0} f^{\prime \prime}+f_{0}^{\prime \prime} f-2 f f_{0}^{\prime}-i \omega f^{\prime}+\frac{\sin \phi}{\zeta} \theta \\
& =\zeta\left(f_{0}^{\prime} \frac{\partial f^{\prime}}{\partial \zeta}+f^{\prime} \frac{\partial f_{0}^{\prime}}{\partial \zeta}-f_{0}^{\prime \prime} \frac{\partial f}{\partial \zeta}-f^{\prime \prime} \frac{\partial f_{0}}{\partial \zeta}\right) \\
& \frac{1}{\operatorname{Pr}} \theta^{\prime \prime}+f_{0} \theta^{\prime}-i \omega \theta+f \theta_{0}^{\prime} \\
& =\zeta\left(f_{0}^{\prime} \frac{\partial \theta}{\partial \zeta}+f^{\prime} \frac{\partial \theta_{0}}{\partial \zeta}-\theta^{\prime} \frac{\partial f_{0}}{\partial \zeta}-\theta_{0}^{\prime} \frac{\partial f}{\partial \zeta}\right)
\end{aligned}
$$

where $\omega$ is the frequency parameter, that depends on the frequency of oscillation of the fluctuating flow.

$$
\begin{aligned}
& f(\zeta, 0)=f^{\prime}(\zeta, 0)=0, \quad \theta(\zeta, 0)=1 \\
& f^{\prime}(\zeta, \infty)=0, \quad \theta(\zeta, \infty)=0
\end{aligned}
$$

One can see the set of equations (22), (23), (25) and (26) together with the boundary conditions (24) and (27) are coupled and hence we need to get the solutions of these equations all at one time to get better results.

\section{METHOD OF SOLUTION}

Now, we are at the position to employ the most efficient and accurate implicit finite difference method together with the Keller-box elimination technique (also known as Keller box method), introduced by Keller [18] and described in more detail in Cebeci and Bradshaw [19] in finding the solutions of the parabolic system of equations (22)-(33) and (25)(26) that represents the steady and oscillatory components of the velocity and temperature fields. To apply the aforementioned method, the system of equations (22)-(23) and (25)(26) are, first, written in terms of a system of first order equations as follows:

$$
\begin{aligned}
& \frac{\partial f_{0}}{\partial \eta}=U_{s}, \frac{\partial U_{s}}{\partial \eta}=V_{s}, \theta_{0}=G_{S}, \frac{\partial \theta_{0}}{\partial \eta}=W_{s} \\
& \frac{1}{\operatorname{Pr}} \frac{\partial V_{s}}{\partial \eta}+p_{1}\left(f_{0} V_{s}-U_{S}^{2}\right)=\zeta\left(U_{s} \frac{\partial U_{s}}{\partial \zeta}-V_{s} \frac{\partial f_{0}}{\partial \zeta}\right) \\
& \frac{1}{\operatorname{Pr}} \frac{\partial W_{s}}{\partial \eta}+p_{1} f_{0} W_{s}=\zeta\left(U_{s} \frac{\partial G_{S}}{\partial \zeta}-W_{s} \frac{\partial f_{0}}{\partial \zeta}\right) \\
& \frac{\partial f_{r}}{\partial \eta}=U_{r}, \frac{\partial U_{r}}{\partial \eta}=V_{r}, \theta_{r}=G_{r}, \theta_{i}=G_{i}, \frac{\partial \theta_{r}}{\partial \eta}=W_{r} \\
& \frac{\partial V_{r}}{\partial \eta}+p_{1}\left(f_{0} V_{r}+f_{r} V_{s}\right)-p_{2} U_{r} U_{s}+p_{4} U_{i}+ \\
& p_{3} G_{r}=\zeta\left(U_{s} \frac{\partial U_{r}}{\partial \zeta}+U_{r} \frac{\partial U_{s}}{\partial \zeta}-V_{s} \frac{\partial f_{r}}{\partial \zeta}-V_{r} \frac{\partial f_{0}}{\partial \zeta}\right) \\
& \frac{1}{\operatorname{Pr}} \frac{\partial W_{r}}{\partial \eta}+p_{1}\left(f_{0} W_{r}+f_{r} W_{s}\right)+p_{4} G_{i}=\zeta \\
& \left(U_{s} \frac{\partial G_{r}}{\partial \zeta}+U_{r} \frac{\partial G_{s}}{\partial \zeta}-W_{r} \frac{\partial f_{0}}{\partial \zeta}-W_{s} \frac{\partial f_{r}}{\partial \zeta}\right) \\
& \frac{\partial f_{i}}{\partial \eta}=U_{i}, \frac{\partial U_{i}}{\partial \eta}=V_{i}, \frac{\partial \theta_{i}}{\partial \eta}=W_{i} \\
& \frac{\partial V_{i}}{\partial \eta}+p_{1}\left(f_{0} V_{i}+f_{i} V_{s}\right)-p_{2} U_{i} U_{s}-p_{4} U_{r}+p_{3} G_{i} \\
& =\zeta\left(U_{s} \frac{\partial U_{i}}{\partial \zeta}+U_{i} \frac{\partial U_{s}}{\partial \zeta}-V_{s} \frac{\partial f_{i}}{\partial \zeta}-V_{i} \frac{\partial f_{0}}{\partial \zeta}\right)
\end{aligned}
$$

The corresponding boundary conditions (20) becomes 


$$
\begin{aligned}
& \frac{1}{\operatorname{Pr}} \frac{\partial W_{i}}{\partial \eta}+p_{1}\left(f_{0} W_{i}+f_{i} W_{r}\right)-p_{4} G_{r}= \\
& \zeta\left(U_{S} \frac{\partial G_{i}}{\partial \zeta}+U_{i} \frac{\partial G_{S}}{\partial \zeta}-W_{i} \frac{\partial f_{0}}{\partial \zeta}-W_{s} \frac{\partial f_{i}}{\partial \zeta}\right)
\end{aligned}
$$

where $p_{1}=1, p_{2}=2, p_{3}=\frac{\sin \phi}{\zeta}$ and $p_{4}=\omega$ then corresponding boundary conditions becomes

$$
\begin{aligned}
& f_{0}(\zeta, 0)=U_{S}(\zeta, 0)=0, G_{S}(\zeta, 0)=1 \\
& U_{S}(\zeta, \infty)=0, G_{S}(\zeta, \infty)=0
\end{aligned}
$$

and

$$
\begin{aligned}
& f_{r}(\zeta, 0)=U_{r}(\zeta, 0)=0, G_{r}(\zeta, 0)=1 \\
& U_{r}(\zeta, \infty)=0, G_{r}(\zeta, \infty)=0 \\
& f_{i}(\zeta, 0)=U_{i}(\zeta, 0)=0, G_{i}(\zeta, 0)=0 \\
& U_{i}(\xi, \infty)=0, G_{i}(\xi, \infty)=0
\end{aligned}
$$

In the above equations functions $f_{r}, f_{r}{ }^{\prime}, f_{r}{ }^{\prime \prime}, \theta_{r}, \theta_{r}{ }^{\prime \prime}$ and $f_{i}$, $f_{i}^{\prime}, f_{i}^{\prime \prime}, \theta_{i}, \theta_{i}^{\prime \prime}$ are the real and imaginary parts of the functions $f, f^{\prime}, f^{\prime \prime}, \theta$ and $\theta^{\prime}$, respectively.

The above equations are then expressed in finite difference form by approximating the functions and their derivatives in terms of the central differences in both co-ordinate directions. Denoting the mesh points in the $(\zeta, \eta)$ plane by $\zeta_{i}$ and $\eta_{j}$, where $i=1,2,3, \ldots, M$ and $j=1,2,3, \ldots, N$, central difference approximations are made such that the equations involving $X$ explicitly are centered at $\left(\xi_{i-1 / 2}, Y_{j-1 / 2}\right)$ and the remainder at $\left(\xi_{i}, \eta_{j-1 / 2}\right)$, where $\eta_{j-1 / 2}=\left(\eta_{j}+\eta_{j-1}\right) / 2$, etc. This results in a set of non-linear difference equations for the unknowns at $\zeta_{i}$ in terms of their values at $\zeta_{i-1}$. These equations are then linearized by the Newton's quasilinearization technique and solved using block-tridiagonal algorithm, introduced by Keller [18] taking as the initial iteration of the converged solution at $\zeta=\zeta_{i-1}$. To initiate the process at $\zeta=0$, we first prescribe the profiles for the functions $f_{0}, f_{0}^{\prime}, f_{0}^{\prime \prime}, \theta_{0}, \theta_{0}^{\prime}$ and $f_{r}, f_{r}^{\prime}, f_{r}^{\prime \prime}, \theta_{r}, \theta_{r}^{\prime \prime}$ and $f_{i}, f_{i}^{\prime}, f_{i}^{\prime \prime}$, $\theta_{i}, \theta_{i}^{\prime \prime}$, from the solutions of the equations (35)-(40) that are obtained numerically using the Newton-Raphson iteration technique together with the sixth-order implicit Runge-Kutta initial value solver.

$$
\begin{aligned}
& f_{0}^{\prime \prime \prime}+f_{0} f_{0}^{\prime \prime}-f_{0}^{\prime 2}+A_{0} \theta_{0}=0 \\
& \frac{1}{\operatorname{Pr}} \theta_{0}^{\prime \prime}+f_{0} \theta_{0}^{\prime}=0 \\
& f_{0}(0)=f_{0}^{\prime}(0)=0, \theta_{0}(0)=1 \\
& f_{0}^{\prime}(\infty)=0, \quad \theta_{0}(\infty)=0
\end{aligned}
$$

$$
\begin{aligned}
& f_{r}^{\prime \prime \prime}+f_{0} f_{r}^{\prime \prime}+f_{r} f_{0}^{\prime \prime}-2 f_{r}^{\prime} f_{0}^{\prime}+\omega f_{i}^{\prime}+A_{0} \theta_{r}=0 \\
& \frac{1}{\operatorname{Pr}} \theta_{r}^{\prime \prime}+f_{0} \theta_{r}^{\prime}+f_{r} \theta^{\prime}+\omega \theta_{i 0}=0 \\
& f_{r}(0)=f_{r}^{\prime}(0)=0, \quad \theta_{r}(0)=1 \\
& f_{r}^{\prime}(\infty)=0, \quad \theta_{r}(\infty)=0 \\
& f_{i}^{\prime \prime \prime}+f_{0} f_{i}^{\prime \prime}+f_{i} f_{0}^{\prime \prime}-2 f_{i}^{\prime} f_{0}^{\prime}-\omega f_{r}^{\prime}+A_{0} \theta_{i}=0 \\
& \frac{1}{\operatorname{Pr}} \theta_{i}^{\prime \prime}+f_{0} \theta^{\prime}+f_{i} \theta_{0}^{\prime}+\omega \theta_{r}=0 \\
& f_{i}(0)=f_{i}^{\prime}(0)=0, \quad \theta_{i}(0)=0 \\
& f_{i}^{\prime}(\infty)=0, \quad \theta_{i}(\infty)=0
\end{aligned}
$$

for pertinent parameters, such as, $\operatorname{Pr}, \omega$ and $A_{0}$.

These profiles are then employed in the Keller box scheme, which has second-order accuracy to march stepwise along the boundary layer. For any given value of $\zeta$, the iterative procedure is stopped to obtain the final velocity and temperature distributions when the difference in computing the velocity, the temperature and the species concentration in the latest iteration is less than $10^{-6}$, i.e., $\Delta f^{i} \leq 10^{-6}$, where the superscript $i$ denotes the number of iterations. Throughout the computations a non-uniform grid has been used by considering $\eta_{\mathrm{j}}=\sinh ((j-1) / \mathrm{d})$, with $j=1,2, \ldots \ldots, 301$ and $d=100$.

Numerical values of the local mean heat transfer coefficient, $-\theta_{0}^{\prime}(\alpha, 0)$, obtained from the above solutions for $\operatorname{Pr}=$ 1.0 with $A_{0}=0.25$ and 0.50 against the eccentric angle $\alpha$, are entered in Tables. 1-2 for two types of cylindrical forms, known as blunt and slender elliptic cylinder. The results are compared and found in excellent agreement with the corresponding solutions obtained by Merkin [2] and Kumar et al. [20].

Now from the set of relation (12) together with the transformation given in (21), we have the following expressions for dimensionless axial velocity and the temperature functions as given below:

$$
\begin{aligned}
& U(\zeta, \eta, \tau)=f_{0}^{\prime}(\zeta, \eta)+\varepsilon\left(\cos \omega \tau f_{i}^{\prime}(\zeta, \eta)-\sin \omega \tau f_{r}^{\prime}(\zeta, \eta)\right) \\
& G(\zeta, \eta, \tau)=\theta_{0}(\zeta, \eta)+\varepsilon\left(\cos \omega \tau \theta_{i}(\zeta, \eta)-\sin \omega \tau \theta_{r}(\zeta, \eta)\right) \quad(46 \mathrm{a}, \mathrm{b})
\end{aligned}
$$

In equation $(46 \mathrm{a}, \mathrm{b}) f_{r}^{\prime}, f_{i}^{\prime}$ and $\theta_{r}, \theta_{i}$ are respectively, the real and imaginary parts of the velocity function, $f^{\prime}(\zeta, \eta)$, and the temperature function, $\theta(\zeta, \eta)$.

The physical quantities that are important from application point of view are the shear stress, $\tau_{\mathrm{w}}$, and the surface rate of heat transfer, $q_{w}$. These can be measured from the non-dimensional relations (47) and (48).

$$
\tau_{w}=\zeta\left[f_{0}^{\prime \prime}(\zeta, 0)+\varepsilon\left|A_{1}\right| \cos \left(\omega t+\alpha_{1}\right)\right]
$$

and 
Table 1 Numerical values of $-\theta_{0}^{\prime}(\alpha, 0)$, for Different Values of $\alpha$ While Pr $=1.0$ and $A_{0}=0.25$, 0.50 for a Blunt Elliptic Cylinder

\begin{tabular}{|c|c|c|c|c|c|c|}
\hline $\mathbf{A}_{0}$ & \multicolumn{3}{|c|}{0.25} & \multicolumn{3}{|c|}{0.50} \\
\hline$\alpha$ & Present IFDM & Kumer et al. [20] & Merkin [02] & Present IFDM & Kumer et al.[20] & Merkin [02] \\
\hline 0.0 & 0.2979 & 0.2980 & 0.2979 & 0.3543 & 0.3543 & 0.3543 \\
\hline 0.2 & 0.2994 & 0.2994 & 0.2994 & 0.3556 & 0.3556 & 0.3556 \\
\hline 0.6 & 0.3120 & 0.3119 & 0.3118 & 0.3659 & 0.3658 & 0.3658 \\
\hline 1.0 & 0.3424 & 0.3420 & 0.3418 & 0.3864 & 0.3863 & 0.3863 \\
\hline 1.4 & 0.4020 & 0.4012 & 0.4008 & 0.4085 & 0.4085 & 0.4084 \\
\hline 1.8 & 0.4062 & 0.4070 & 0.4070 & 0.3954 & 0.3959 & 0.3959 \\
\hline 2.2 & 0.3231 & 0.3238 & 0.3241 & 0.3399 & 0.3406 & 0.3406 \\
\hline 2.6 & 0.2467 & 0.2473 & 0.2476 & 0.2744 & 0.2750 & 0.2750 \\
\hline 3.0 & 0.1780 & 0.1788 & 0.1791 & 0.2046 & 0.2054 & 0.2054 \\
\hline$\pi$ & 0.1501 & & 0.1504 & 0.1747 & & 0.1746 \\
\hline
\end{tabular}

Table 2. Numerical Values of the $-\theta_{0}^{\prime}(\alpha, 0)$ (Mean Flow)for Different Values of $\alpha$ While $\operatorname{Pr}=1.0$ and $b / a=0.25$ fora Slender Elliptic Cylinder

\begin{tabular}{|c|c|c|c|}
\hline $\boldsymbol{\alpha}$ & Present IFDM & Kumer et al. [20] & Merkin [02] \\
\hline \hline 0.0 & 0.8426 & 0.8428 & 0.8359 \\
0.2 & 0.7706 & 0.7722 & 0.7682 \\
0.4 & 0.6619 & 0.6632 & 0.6617 \\
0.6 & 0.5781 & 0.5794 & 0.5788 \\
0.8 & 0.5175 & 0.5191 & 0.5187 \\
1.0 & 0.4729 & 0.4747 & 0.4745 \\
1.2 & 0.4392 & 0.4410 & 0.4409 \\
1.4 & 0.4132 & 0.4150 & 0.4149 \\
1.6 & 0.3929 & 0.3944 & 0.3943 \\
1.8 & 0.3768 & 0.3779 & 0.3779 \\
2.0 & 0.3641 & 0.3646 & 0.3646 \\
2.2 & 0.3538 & 0.3537 & 0.3538 \\
2.4 & 0.3451 & 0.3446 & 0.3447 \\
2.6 & 0.3370 & 0.3362 & 0.3363 \\
2.8 & 0.3270 & 0.3262 & 0.3266 \\
3.0 & 0.3062 & 0.3070 & 0.3084 \\
$\pi$ & 0.2780 & & 0.2785 \\
\hline
\end{tabular}

$q_{w}=-\left[\theta_{0}^{\prime}(\zeta, 0)+\varepsilon\left|A_{2}\right| \cos \left(\omega t+\alpha_{2}\right)\right]$

Here prime denote differentiation with respect to $\eta$ and $\left|A_{1}\right|$ and $\left|A_{2}\right|$ are the amplitudes and the $\alpha_{1}$ and $\alpha_{2}$ are the phase angles, respectively, for the local skin friction and the local heat transfer for the fluctuating flow and temperature field which readily available from the following relations:

$\left|A_{1}\right|=\left({f_{r}^{\prime \prime}}^{2}+{f_{i}^{\prime \prime}}^{2}\right)^{1 / 2}, \quad\left|A_{2}\right|=\left({\theta_{r}^{\prime}}^{2}+{\theta_{i}^{\prime}}^{2}\right)^{1 / 2}$

and $\alpha_{1}=\tan ^{-1}\left(\frac{f_{i}^{\prime \prime}}{f_{r}^{\prime \prime}}\right), \quad \alpha_{2}=\tan ^{-1}\left(\frac{\theta_{i}^{\prime}}{\theta_{r}^{\prime}}\right)$

where $f_{r}^{\prime \prime}, f_{i}^{\prime \prime}$ and $\theta_{r}^{\prime}, \theta_{i}^{\prime}$ are respectively the real and imaginary parts of the coefficients $f^{\prime \prime}(\zeta, 0)$ and $\theta^{\prime}(\zeta, 0)$.

Numerical vales of the amplitudes, $\left|A_{1}\right|$ and $\left|A_{2}\right|$, and the phase-angles, $\alpha_{1}$ and $\alpha_{2}$, of the oscillating shear stress and heat transfer rate, respectively, are obtained for different values of the pertinent physical parameters, $\omega$, the frequency parameter, Pr, the Prandtl number and $A_{0}$, against eccentric angle $\alpha$. The results presented and discussed in the following section are based on the solution obtained by the above methods.

It's worth mentioning that, the present problem for the case $a=b$ (circular cylinder) represents the oscillatory flow along circular cylinders that has recently been discussed by Jaman et al. [17].

\section{RESULTS AND DISCUSSION}

Consideration is hereby given to the implicit finite difference method being employed in finding the solutions of the equations that govern the oscillating free convection flow along a heated blunt and slender elliptic cylinder, for different values of $\omega$, the frequency parameter, $\varepsilon$, amplitude of oscillation, and various values of $A_{0}$ for a fluid having the value of Prandtl number, $\operatorname{Pr}=0.1$. Although the quantities such as surface heat flux and shear stress are very important, here we present only the surface heat flux to compare with those of other authors for the steady state flow.

We begin by representing some results obtained from the present analysis for elliptic cylinders since experimental data and results from other authors are available for comparison. The rate of heat transfer coefficient, $-\theta_{0}^{\prime}(\alpha, 0)$ are obtained for different values of the physical parameters $A_{0}=0.25$, 0.50 and $\operatorname{Pr}=1.0$ which are summarized in Table 1 against eccentric angle $\alpha$ in the interval $[0, \pi]$. In this table we also 
compare the results with that obtained by Merkin [2], Kumer et al. [20] for the blunt cylinders. In Table 2, numerical values of the $-\theta_{0}^{\prime}(\alpha, 0)$ (mean flow) for different values of $\alpha$ while $\operatorname{Pr}=1.0$ and $b / a=0.25$ for a slender elliptic cylinder being compared with obtained the above mentioned authors. Considering all factors, it seems reasonable to conclude that the agreement is good as one may hope for.

The implicit finite difference method together with Keller-box elimination scheme for the entire range of frequency are employed in finding the solutions of the equations governing the unsteady free convection flow along uniformly heated blunt and slender elliptic cylinders.

The foregoing coupled differential equations (25) and (26), representing the fluctuating parts of the flow and temperature fields together with the boundary conditions (27) have, numerically, been integrated by the methodology discussed above. We can see that the fluctuating parts of the flow and the temperature fields are not only dependent on the eccentric angle but also on the other physical quantities, like the Prandtl number, Pr, and the frequency of oscillation on the surface temperature, $\omega$, the amplitude of oscillation, $\varepsilon$. Thus obtained the simulated results are displayed in terms of amplitude and phase of the skin-friction, $\tau_{x}$, and the rate of heat transfer, $q_{x}$, for values of the ratio of the semi-minor and semi-major axis for both the blunt and slender bodies, $b / a=0.5,0.75$ and 1.0 , for fluid having Prandtl number, $\mathrm{Pr}=0.1$. Effects of the aforementioned parameters are discussed in details in the following paragraphs.

The numerical values of the amplitude and phase, $\left(\left|A_{1}\right|\right.$, $\alpha_{1}$ ), of the local shear stress and that of rate of heat transfer, $\left(\left|A_{2}\right|, \alpha_{2}\right)$, for the fluctuating flow are depicted in Figs. (2-5) for both blunt and slender bodies. We shall embody from presenting the details.

\section{Effect of Physical Parameters on Amplitude and Phase of Skin-Friction and Heat Transfer}

The numerical values of the amplitude and phase, $\left(\left|A_{1}\right|\right.$, $\alpha_{1}$ ), of the local shear stress and that of rate of heat transfer, $\left(\left|A_{2}\right|, \alpha_{2}\right)$, for the fluctuating flow are depicted in Figs. (2-5) for both blunt and slender bodies. We shall embody from presenting the details.

For blunt and slender orientation, numerical values of amplitude, $\left|A_{1}\right|$, for shear stress are shown, respectively, in Figs. (2a) and (3a) for values of $b / a=0.5,0.75,1.0$ while $\omega=0.5$ and $\omega \tau=\pi / 4$. From these Figures one can see that the amplitude, $\left|A_{1}\right|$, of the shear stress for both the cylindrical (a)

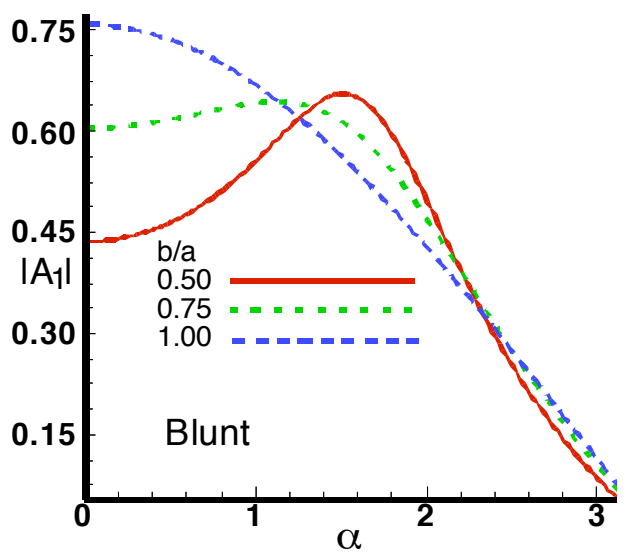

(b)

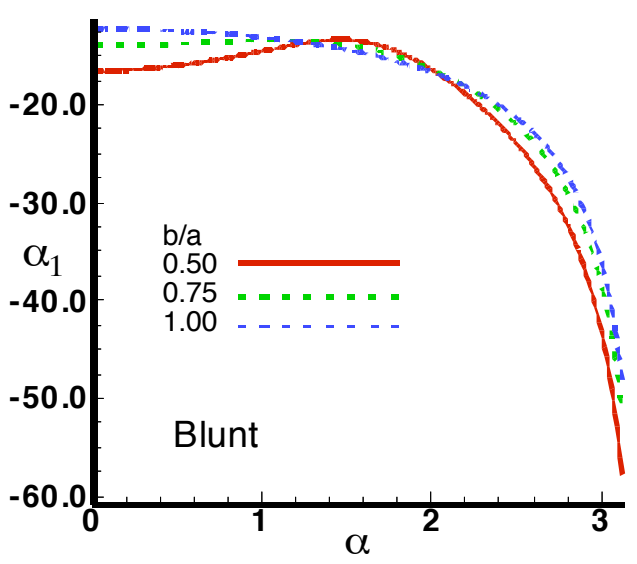

Fig. (2). Amplitude and phase of skin-friction from a blunt surface at $\operatorname{Pr}=0.1, \omega=0.5, \omega \tau=\pi / 4$ while $\mathrm{b} / \mathrm{a}=0.50,0.75$ and1.0.

(a)

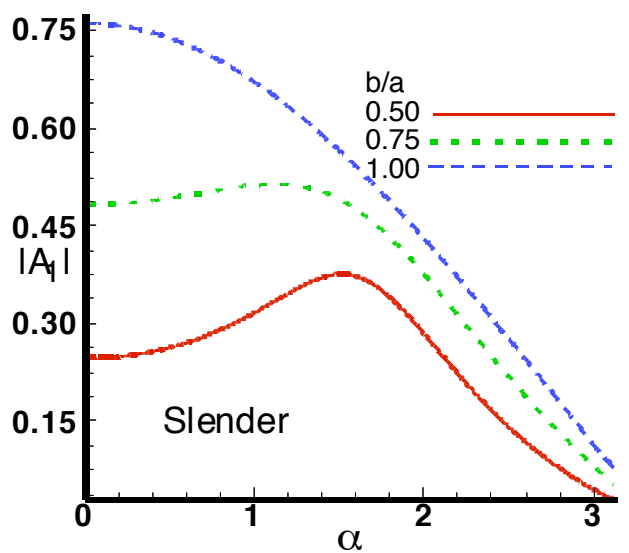

(b)

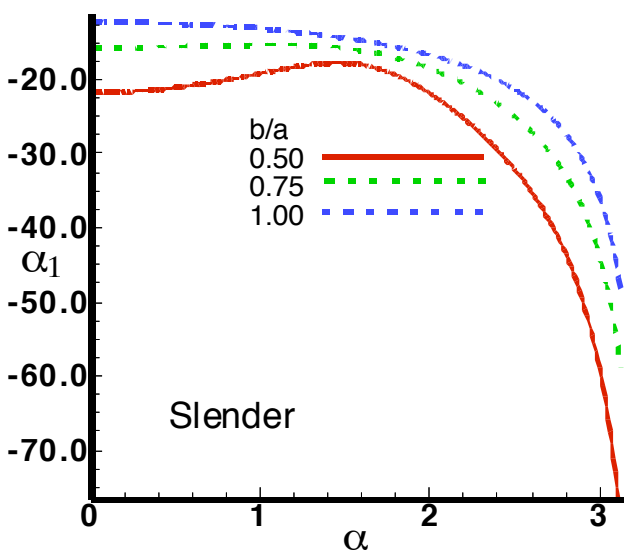

Fig. (3). Amplitude and phase of skin-friction from a slender surface at $\operatorname{Pr}=0.1, \omega=0.5, \omega \tau=\pi / 4$ while $\mathrm{b} / \mathrm{a}=0.50,0.75$ and 1.0 . 
(a)

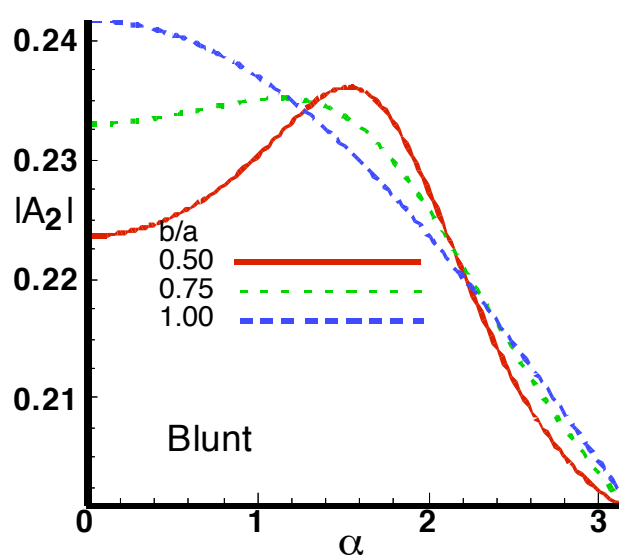

$\alpha$

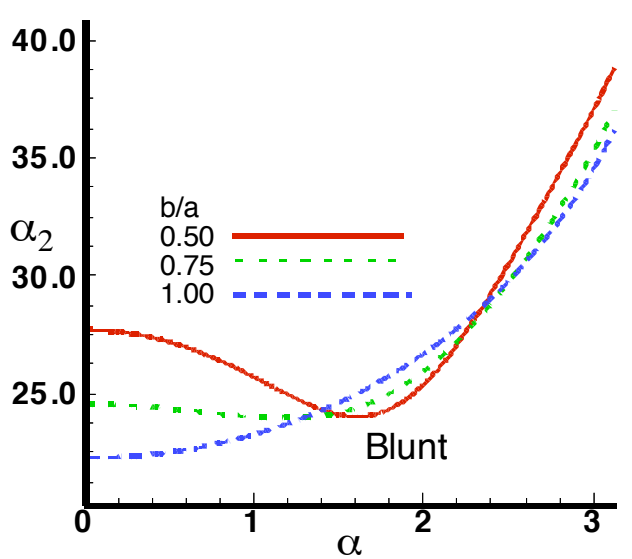

$\alpha$

Fig. (4). Amplitude and phase of heat transfer from a blunt surface at $\operatorname{Pr}=0.1, \omega=0.5, \omega \tau=\pi / 4$ while $b / a=0.50,0.75$ and 1.0 .

(a)

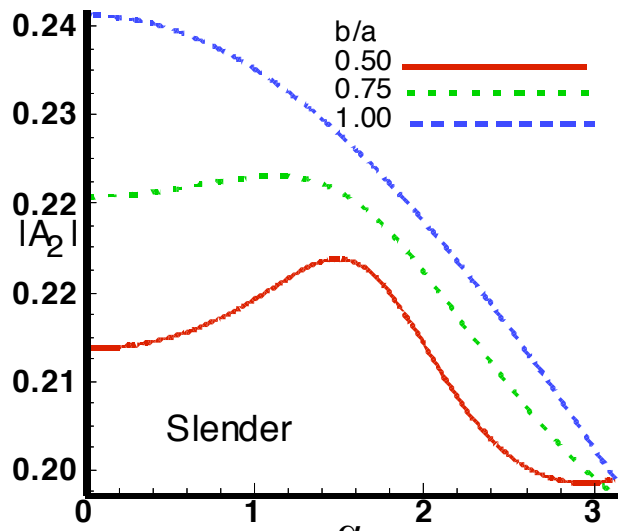

$\alpha$ (b)

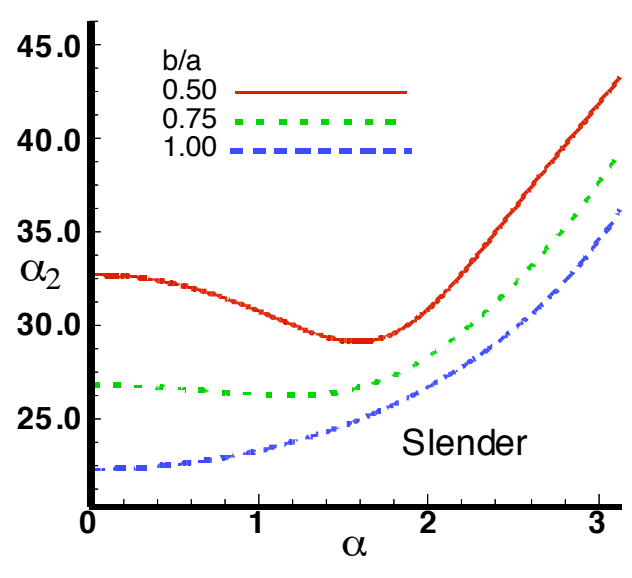

circular and graphically blunt and slender body represents the identical manner.

The variation of the amplitude and the phase of the fluctuating surface rate of heat transfer over the periphery of elliptical cylinders of aspect ratio $b / a=0.5,0.75$ and 1.0 are shown in Figs. (4-5). It can be seen from Figs. (4a), (5a) that the amplitude $\left|A_{2}\right|$, of the fluctuating rate of heat transfer for blunt ellipse increases initially with the increase of the eccentric angle $\alpha$ and then it leads to decrease; on the other hand, for slender ellipse the value of amplitude of the skin friction has alike manner as for blunt body. For increasing the fractional values of $b / a$, initially amplitude is increasing and then decreasing in haste for blunt surface and constantly decrease for other surface. We further notice that the relative maximum value of the amplitude occurs at the lower stagnation point for both cylinder surfaces.

In Figs. (4b), (5b), the computed phase of fluctuating rate of heat transfer are plotted for elliptic cylinders over a Prandtl number $\operatorname{Pr}=0.1$ in the effect of the partial values of $b / a$. One finds that phase of oscillation $\alpha_{2}$ in the fluctuating rate of heat transfer are decreasing for $A_{0}=0.50$ upto the eccentric angle $\alpha=1.8$ radian and then it start to increase for blunt body and similar approach shows the slender body. In favor of increasing values of $b / a$ phase angle is decreasing in mixing order for blunt surface and continuously decreasing for slender surface. Continually there is a phase lead for both cylinders. In the above discussion for amplitude and phase may be available for comparison but we mention that as the parameter $A_{0}=1$, in that case both type of cylinders becomes 
for different cylindrical surface, we conclude that both surfaces represent the similar behaviors when they divert from circular cylinder.

\section{Effect of Physical Parameter on Streamlines and Isotherms}

Now we see the effects of physical parameters, such as, $b / a$ and $\omega$, controlling the present problem on the flow pattern and the temperature distribution in terms of the streamlines and isotherms in the boundary layer regime for both blunt and slender cylinder through Figs. (6 to 9). Following relations are considered to measure the values of oscillating stream-function and the oscillating temperature in the boundary layer regime:

$\psi(\zeta, \eta, \tau)=\xi\left(f_{0}(\zeta, \eta)+\varepsilon\left(\begin{array}{l}\left.f_{i}(\zeta, \eta) \cos \omega \tau+\right) \\ f_{r}(\zeta, \eta) \sin \omega \tau\end{array}\right)\right)$

and

$\theta(\zeta, \eta, \tau)=\theta_{0}(\zeta, \eta)+\varepsilon\left(\begin{array}{l}\theta_{i}(\zeta, \eta) \cos \omega \tau+ \\ \theta_{r}(\zeta, \eta) \sin \omega \tau\end{array}\right)$

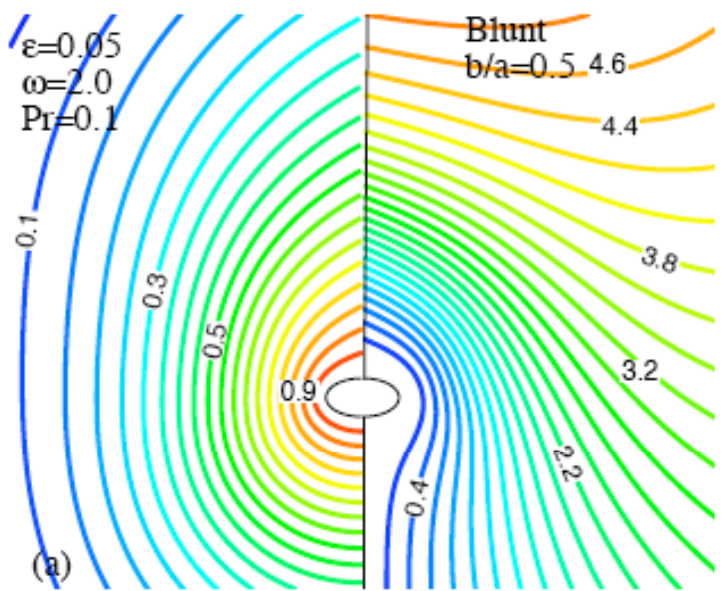

In these figures, the left column of graphs isotherms are shown and in the right column streamlines. Numerical solutions are obtained for different eccentricity, $e$ while $\omega \tau=\pi / 4$ and $\varepsilon=0.05$ and for a unit value of Prandtl number.

A comparisons of streamlines and isotherms for values of $b / a=1.0,1 / 2$ and $1 / 3$ are shown in Figs. (6 and 7). From these figures one can observe that there is relatively little change in the streamlines for slender body but conspicuous changes happens for blunt body while the eccentricity increases. Because of increase of the eccentricity value of $\psi$ decreases for blunt cylinder (having $\psi_{\max }=5.20$ ); on the other hand its value increases in case of slender cylinder (for which $\left.\psi_{\max }=5.57\right)$. This implies that for blunt surface, the momentum boundary layer becomes thinner and the flow is weaker and for slender surface it becomes thicker and the flow becomes stronger because of increasing eccentricity of the cylinders. From the given isotherms one can see that, the fluid temperature is higher near the lower boundary and lower near the upper boundary and this is the region of lowest viscosity. Nearly parallel isotherms in the upper part of the cell indicate that heat transport is almost entirely by conduction.

Figs. (8 and 9) depict the isotherms and streamlines in the same model for the values of $\omega=0.0,1.0$ and 2.0 while

Fig. (6). Isotherms and streamline along blunt elliptic cylinder at $\tau=10$ while $\omega=2.0, \operatorname{Pr}=0.1$ and $\varepsilon=0.05$ for $(\mathbf{a}) \mathrm{b} / \mathrm{a}=0.25$ and (b) $\mathrm{b} / \mathrm{a}=0.5$.
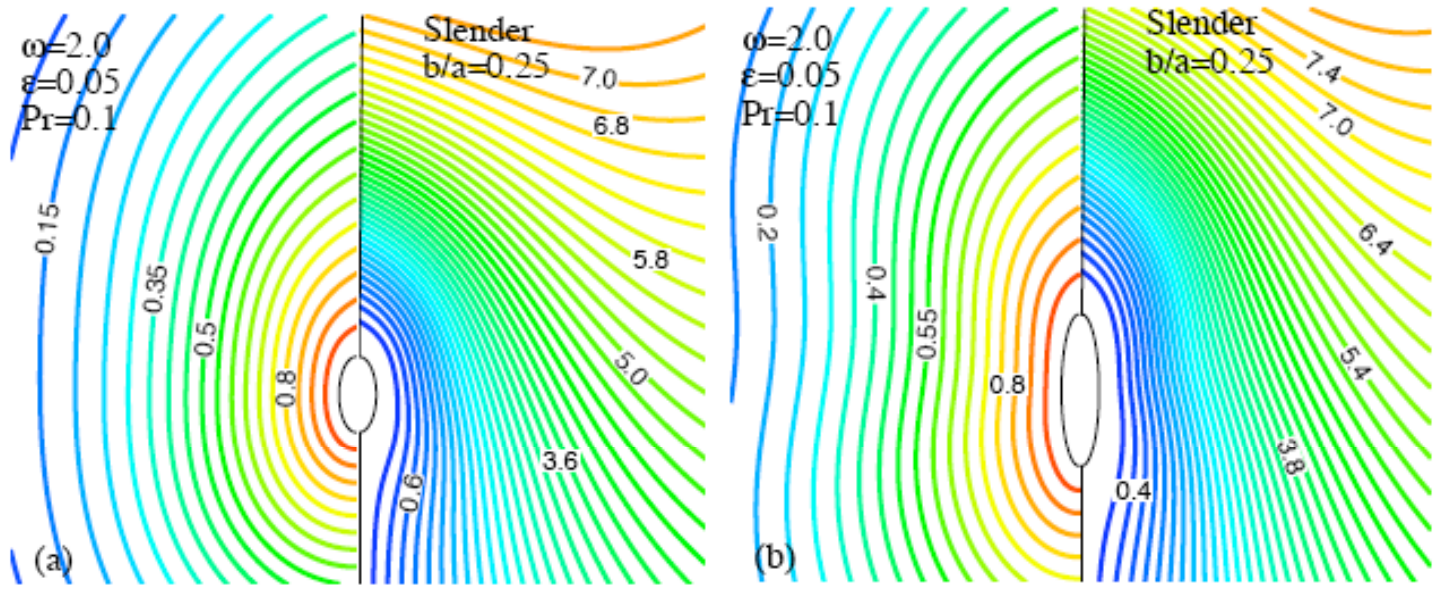

Fig. (7). Isotherms and streamline along slender elliptic cylinder at $\tau=10.0$ while $\omega=2.0, \operatorname{Pr}=0.1$ and $\varepsilon=0.05$ for $(\mathbf{a}) \mathrm{b} / \mathrm{a}=0.25$ and $(\mathbf{b}) \mathrm{b} / \mathrm{a}=0.5$. 

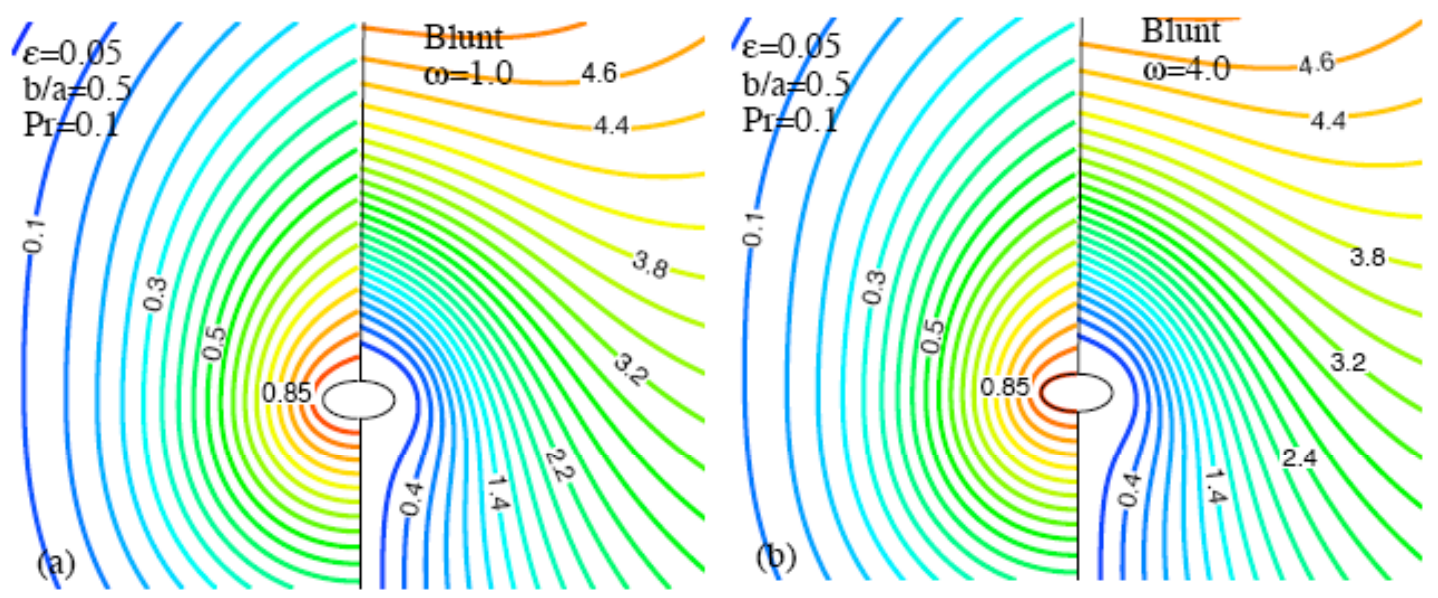

Fig. (8). Isotherms and streamline along blunt elliptic cylinder at $\tau=20$ while $\alpha=\pi / 2, \operatorname{Pr}=0.1, \varepsilon=0.05$ and $\mathrm{b} / \mathrm{a}=0.5:(\mathbf{a}) \omega=1.0$ and $(\mathbf{b}) \omega=4.0$.
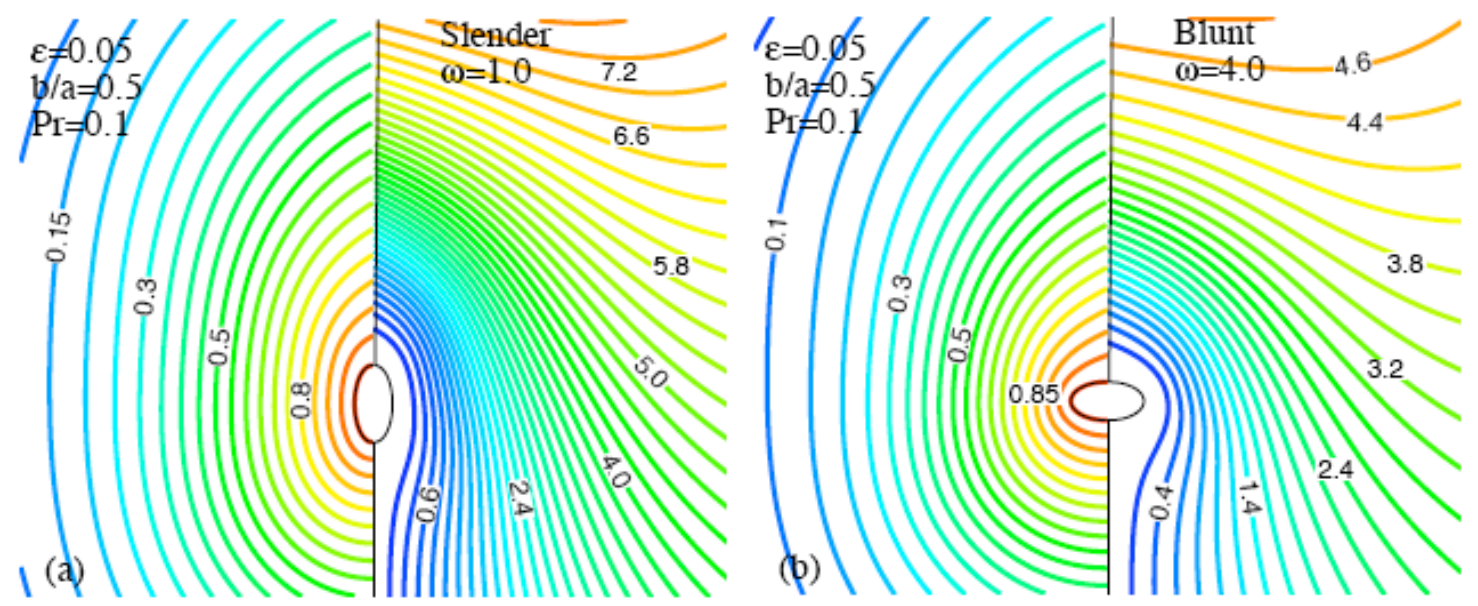

Fig. (9). Isotherms and streamline along slender elliptic cylinder at $\tau=20$ while $\alpha=\pi / 2, \operatorname{Pr}=0.1, \varepsilon=0.05$ and $\mathrm{b} / \mathrm{a}=0.5$ : (a) $\omega=1.0$ and (b) $\omega=4.0$.

$b / a=0.5$. In this case it is found that for blunt surface $\psi_{\max }=3.77$ and for slender surface $\psi_{\max }=5.50$. For both cylinders the viscosity is minimum at the upper boundary and maximum at the lower boundary. For $\omega=0.0$, the momentum boundary layer becomes higher and we have a stronger flow, on the other hand, for increasing the frequency of oscillation i.e., for $\omega=1.0$ and $\omega=2.0$, the momentum boundary layer becomes thinner and we have a weaker flow in the downstream region. This case is designed mutually for blunt and slender orientations. From these figures one can also see that, the thermal boundary layer become thinner with the increasing value of $\omega$.

\section{Effect of Physical Parameter on Transient Shear Stress and Heat-Transfer}

Numerical values of the transient shear stress, $\tau_{w}$, and heat-transfer, $q_{w}$, against $\tau$ obtained from the expressions (47) and (48), which are shown graphically in figures through (10)-(13). Effect of the frequency parameter, $\omega$ and the values of $b / a$ on the development of transient skinfriction and heat-transfer coefficients, for $\operatorname{Pr}=0.1, \omega \tau=\pi / 4$ and $\varepsilon=0.05$ at $\phi=\pi / 2$, are shown in Figs. (10 and 13). Here numerical values of the oscillating skin-friction and heattransfer coefficient against the dimensionless time variable $\tau$ have been shown in Figs. (10 and 11) for values of $\omega=1.0$,
2.0 and 3.0. From these figures, it is observed that at every station of $\tau$, owing to increase in the value of $\omega$, for both the blunt and slender cylinders, there is a decrease in the amplitude of oscillation in the fluctuating shear stress and an increase in the fluctuating heat transfer coefficients. But there is decrease in the phase of oscillation in both phases coefficients due to skin friction and heat transfer. This is expected since increase of frequency of the surface temperature should lead to increase the frequency of oscillation of the shear-stress and temperature of the fluid in the vicinity of the surface of the cylinders.

Now we are looking into the effect of change in bluntness and slenderness on the fluctuating skin-friction and heat transfer from the surface. Effect of these geometric changes taking the value of $b / a$ to be $0.25,0.5$ and 0.75 on the skin-friction and heat transfer are shown, respectively, in Figs. (12 and 13). In this regards $\operatorname{Pr}=0.1, \omega=2.0$ and $\varepsilon=0.05$ at $\phi=\pi / 2$ have been taken. In Fig. (12), one can see that when the value of $b / a$, is increased magnitude of the skin friction get increased for blunt cylinder and decreased for slender cylinder at every $\tau$ station. The rate of increase in the magnitude of the skin-friction for slender cylinder with the increase of $b / a$ considerably is higher than that of blunt cylinder. 

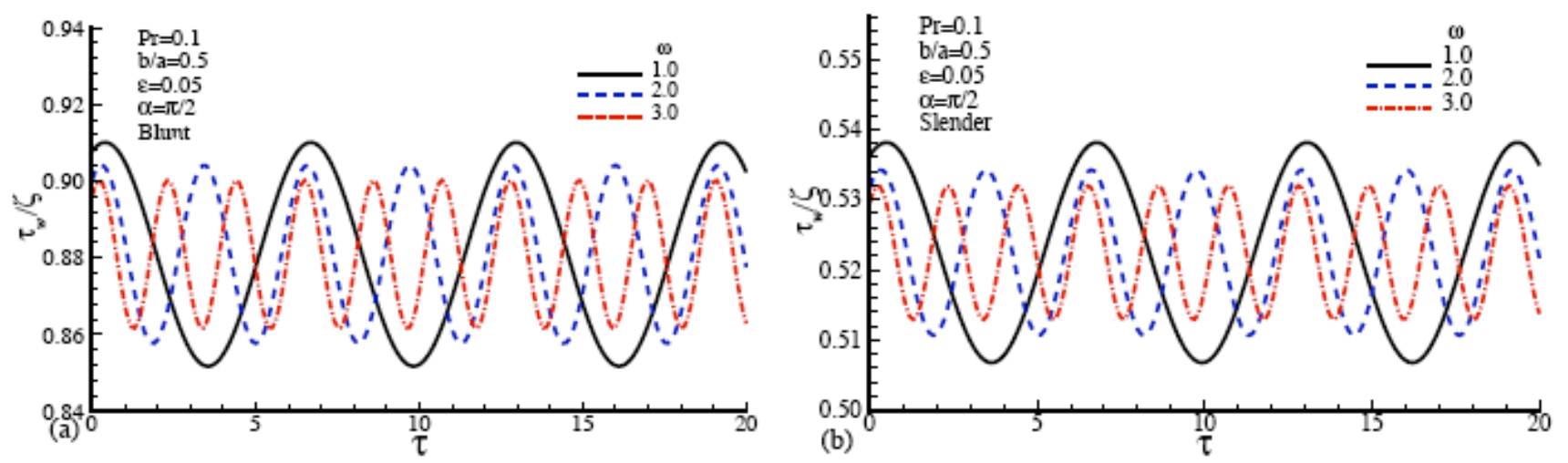

Fig. (10). Transient skin-friction coefficient against $\tau=20$ at $\alpha=\pi / 2, \operatorname{Pr}=0.1, \mathrm{~b} / \mathrm{a}=0.5, \varepsilon=0.05$ for $\omega=1.0,2.0$, and 3.0 for (a) blunt elliptic cylinder (b) slender elliptic cylinder.
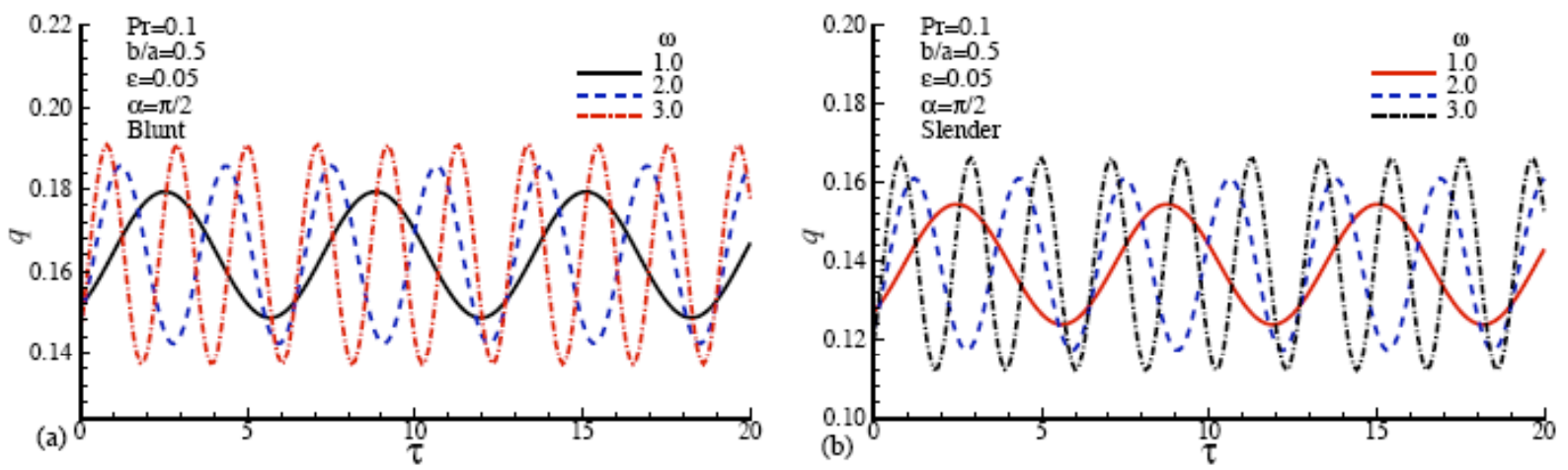

Fig. (11). Transient heat-transfer coefficient against $\tau=20$ at $\alpha=\pi / 2, \operatorname{Pr}=0.1, \mathrm{~b} / \mathrm{a}=0.5, \varepsilon=0.05$ for $\omega=1.0,2.0$, and 3.0 for (a) blunt elliptic cylinder (b) slender elliptic cylinder.
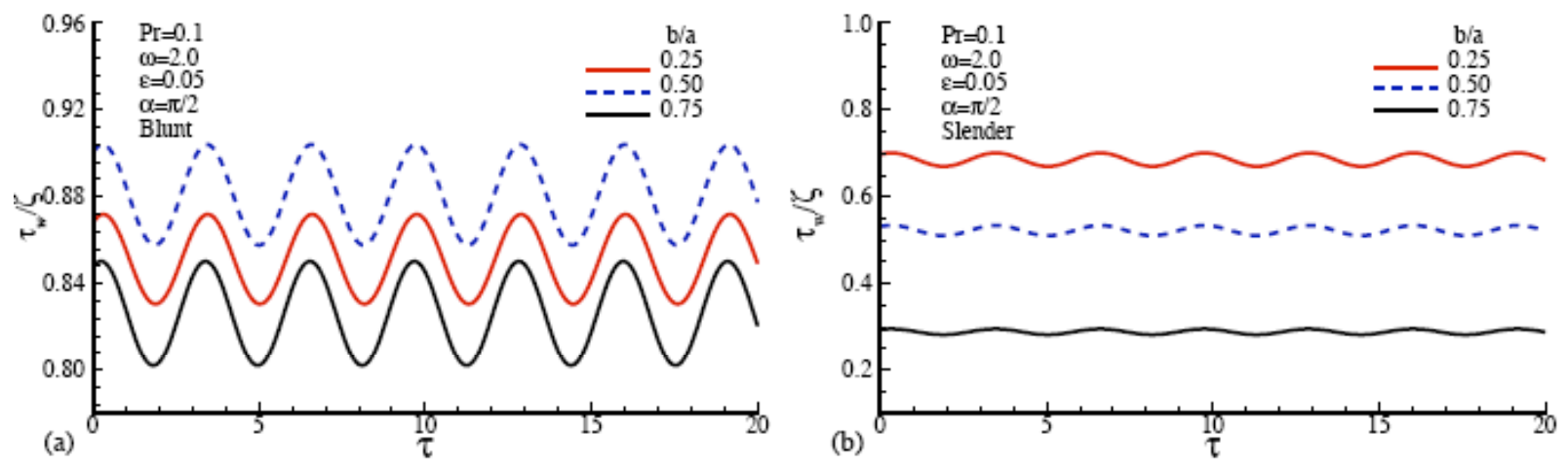

Fig. (12). Transient skin-friction coefficient against $\tau=20$ at $\alpha=\pi / 2, \operatorname{Pr}=0.1, \omega=2.0, \varepsilon=0.05$ for $b / a=0.25,0.5,0.75$ for (a) blunt elliptic cylinder (b) slender elliptic cylinder.

Finally, from the numerical values of the fluctuating surface heat-transfer coefficient, obtained for values of $b / a=$ 0.50, 0.75 and 1.0 shown in Fig. (13) that the amplitude of oscillation of the surface heat-flux has a small change for both kind of the cylinders. The comparison between the three curves for $b / a=0.25,0.5$ and 0.75 , it can be seen that increase in the values of $b / a$ leads to increase in the magnitude of the surface heat flux, $q_{w}$, for both the blunt and slender cylinders.

\section{CONCLUSIONS}

The investigation carried out here is concerned with twodimensional oscillatory natural convection boundary-layer flow of a viscous incompressible fluid past an elliptic cylin- der. Using the appropriate transformation, we have derived the governing equations in the form of stream-function formulation (SFF) and the free variable formulation (FVF). The equations of SFF are then integrated numerically employing the finite difference method together with Keller-box elimination technique and that of FVF by the direct finite difference method together with Gauss elimination technique. The steady state problem that was investigated by Merkin [2] and Kumar et al. [20] has been revisited by the aforementioned methods. It is found that our results are in excellent agreement with these authors.

The results of oscillating flow have been obtained in terms of amplitude and phase of local skin friction and rate of heat transfer with the effect of the physical parameters, 

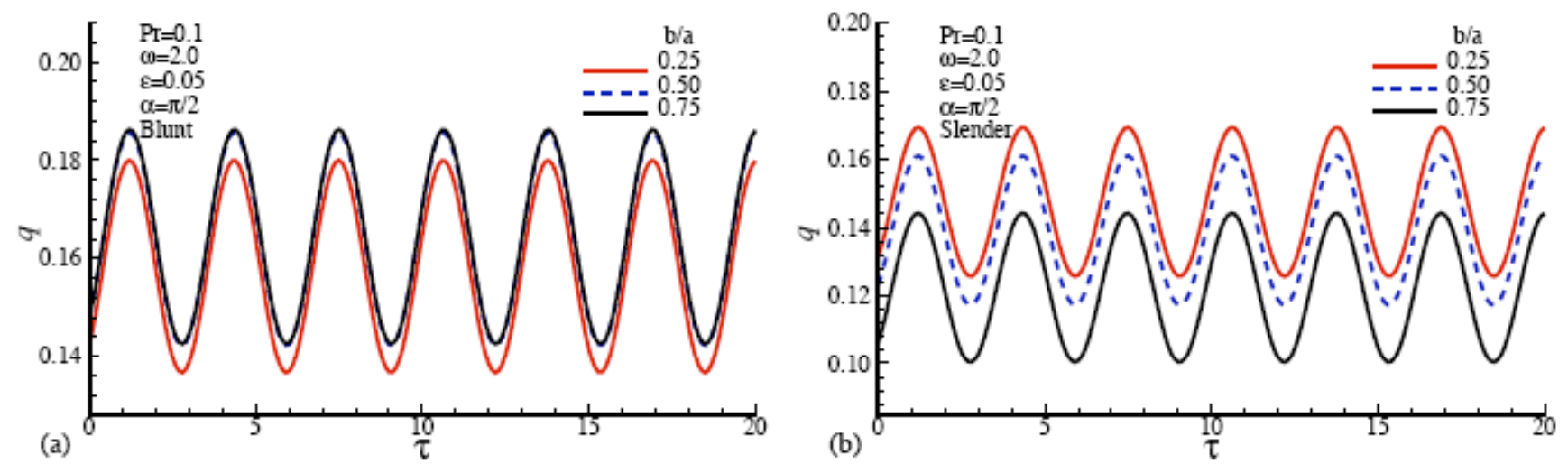

Fig. (13). Transient heat-transfer coefficient against $\tau=20$ at $\alpha=\pi / 2, \operatorname{Pr}=0.1, \omega=2.0, \varepsilon=0.05$ for $b / a=0.25,0.5,0.75$ for (a) blunt elliptic cylinder (b) slender elliptic cylinder.

namely, $\operatorname{Pr}, \omega, \varepsilon$ and $b / a$. Effects of the same parameters are also shown on the fluctuating shear stress and surface rate of heat transfer as well as on the fluctuating streamlines and isotherms. The conclusions that may be taken from the present numerical solutions are given below:

1. For an increase of the numerical values of $b / a$, the amplitude of skin friction increase for blunt and slender surface. Amplitude of the skin-friction for both the blunt and slender cylinders are strictly decreasing when $b / a=1.0$.

2. The phase of oscillation in the skin-friction, $\alpha_{1}$ decrease owing to increase in the ratio $b / a$ with the distance of the eccentric angle $\alpha$ for slender cylinder. For blunt cylindrical surface this trend of $\alpha_{1}$ decreases with the increase of eccentric angle and there is a phase lag. For both type of cylinder the phase $\alpha_{1}$ decreases faster in the downstream region near the upper stagnation point.

3. There is always lag in the phase, $\alpha_{1}$ of the skin friction for blunt and slender surface. Whereas, the phase, $\alpha_{2}$ of the local rate of heat transfer is always in lead for both type of cylinders.

4. Amplitude, $\left|A_{1}\right|$ of the skin friction and $\left|A_{2}\right|$ of the rate of heat transfer for blunt and slender orientation increase owing to increasing value of $b / a$.

5. The fluid temperature is highest near the lower boundary and decreasing to the upper boundary and this is the region of lowest viscosity.

6. For blunt body the momentum boundary layer becomes thinner and have a weaker flow, on the other hand for slender body it becomes thicker and have a stronger flow intended for decreasing the values of $b / a$.

7. There is a decrease in the amplitude of oscillation in the fluctuating shear stress and an increase in the fluctuating heat transfer coefficients owing to increase in the frequency of oscillating surface temperature.

\section{ACKNOWLEGEMENT}

The authors would like to express their gratitude to the reviewers for their positive suggestions for the improvement of quality of this manuscript.

\section{NOMENCLATURE}

\begin{tabular}{|c|c|c|}
\hline$a$ & $=$ & semi-major axis of the cylinder \\
\hline$b$ & $=$ & semi-minor axis of the cylinder \\
\hline$A_{0}$ & $=$ & constant define in Eq. (10) and (11) \\
\hline$e$ & $=$ & eccentricity of the cylinder \\
\hline$C_{p}$ & $=$ & specific heat at constant pressure \\
\hline$f$ & $=$ & dimensionless stream function \\
\hline$g$ & $=$ & acceleration due to gravity \\
\hline $\mathrm{Gr}$ & $=$ & Grashof number \\
\hline $\operatorname{Pr}$ & $=$ & Prandtl number \\
\hline$q_{\mathrm{w}}$ & $=$ & surface rate of heat transfer \\
\hline$T$ & $=$ & temperature of the fluid \\
\hline$T_{w}$ & $=$ & temperature of the heated surface \\
\hline$T_{\infty}$ & $=$ & temperature of the ambient fluid \\
\hline$G$ & $=$ & dimensionless temperature function \\
\hline$G_{0}$ & $=$ & mean temperature \\
\hline$u, v$ & $=$ & $\begin{array}{l}\text { the dimensional } x \text { and } y \text { component of the ve- } \\
\text { locity }\end{array}$ \\
\hline$U, V$ & $=$ & $\begin{array}{l}\text { the dimensionless } X \text { and } Y \text { component of the } \\
\text { velocity }\end{array}$ \\
\hline$x, y$ & $=$ & $\begin{array}{l}\text { coordinates measuring distance round and nor- } \\
\text { mal to the cylinder }\end{array}$ \\
\hline$t$ & $=$ & time \\
\hline
\end{tabular}

\section{Greek Symbol}

$\alpha=$ eccentric angle

$\beta=$ coefficient of thermal expansion

$\kappa=$ thermal conductivity

$\mu \quad=\quad$ dynamic viscosity

$v \quad=\quad$ kinematic viscosity

$\varphi \quad=\quad$ angle made by the outward normal from the cylinder 


$\phi \quad=\quad \begin{aligned} & \text { the angle made by the outward normal from } \\ & \text { the cylinder }\end{aligned}$
$\theta \quad=\quad$ dimensionless temperature function
$\rho \quad=\quad$ density of the fluid
$\varepsilon \quad=$ amplitude of oscillation
$\omega \quad=\quad$ frequency of oscillation
$\psi \quad=$ stream function
$\tau \quad=$ dimensionless time
$\tau_{\mathrm{w}}=$ shear stress
$\eta \quad=\quad$ similarity variable
$\zeta \quad=\quad$ dimensional distance measured along the

\section{Subscripts \& Superscripts}

\section{Surface Conditions}

$$
\begin{array}{lll}
w & = & \text { ambient temperature } \\
\infty & = & \text { differentiation with respect to } \eta
\end{array}
$$

\section{REFERENCES}

[1] J. H. Merkin, "Oscillatory free convection from an infinite horizontal cylinder", J. Fluid Mech., vol. 30, pp. 561-576, 1967.

[2] J. H. Merkin, "Free convection boundary layers on cylinders of elliptic cross section", J. Heat Transf., vol. 99, pp. 453-457, 1977.

[3] M. A. Hossain, and M. A. Alim, "Effect of thermal radiation on natural convection over cylinders of elliptic cross section", Acta Mechanica, vol. 129, pp. 177-186, 1998.

[4] D. A. Saville, and S. W. Churchill, "Laminar free convection in boundary layers near horizontal cylinders and vertical bodies", $J$. Fluid Mech., vol. 29, pp. 391-399, 1967.

[5] F. N. Lin, and B. T. Chao, "Laminar free convection over two-dimensional and axisymmetric bodies of arbitrary contours", J. Heat Transf. Trans. ASMF. Ser. C, vol. 96, pp. 435-442, 1974.
[6] M. J. Lighthill, "The response of laminar skin friction and heat transfer to fluctuations in the stream velocity", Proc.Roy. Soc. London, Ser. A, vol. 224, pp. 1-23, 1954.

[7] M. B. Glauert, and M. J. Lighthill, "The axisymmetric boundary layer on a long thin cylinder", Proc.Roy. Soc. London, Ser. A, vol. 230, pp. 188-203, 1995.

[8] S. Gibellato, "Strato limito attorno ad una lastra piana investita da un fluido in-compressibile dotato di una velocita' che e' soma di una parte constante e di una parte alternata", Atti Accad. Torino, vol. 89-90, pp. 1, 180, 1995.

[9] A. Ghosh, "Contribution a l'etude de la couche limite laminaire instationnaire", Publ. Sci, de la Ministe're de l'Air, Report no. 381, 1961.

[10] R. L. Verma,: "Free convection fluctuating boundary layer on a horizontal plate“. J. Appl. Math. Mech., vol. 63, pp. 483-487, 1982.

[11] R. S. Nanda, and V. P. Sharma, "Free convection laminar boundary layers in oscillatory flow", J. Fluid Mech., vol. 15, pp. 419-428, 1963.

[12] G. M. Harpole, and L. Catton, "Laminar natural convection about downward facing heated blunt bodies of liquid metals", J. Heat Transf., vol. 76-HT-HH, pp. 208-212, 1976.

[13] M. D. Kelleher, and K. T. Yang, "Heat transfer response of laminar free convection boundary layers along a vertical heated plate to surface-temperature oscillations", J. Appl. Math. Phys., vol. 19, pp. 3144, 1968.

[14] S. Eshghy, V. S. Arpaci, and J. A. Clark, "The effect of longitudinal oscillation on free convection from vertical surface", J. Appl. Mech., vol. 32, pp. 183-191, 1965.

[15] M. A. Hossain, S. Hussain, and D. A. S. Rees, "Influence of fluctuating surface temperature and concentration on natural convection flow form a vertical flat plate", J. Appl. Math. Mech., vol. 81, pp. 699-709, 2001.

[16] M. A. Hossain, S. K. Das, and I. Pop, "Heat transfer response of MHD free convection flow along a vertical plate to surface temperature oscillations", Int. J. Non-Linear Mech., vol. 23, pp. 541-553, 1998.

[17] M. K. Jaman, and M. A. Hossain, "Fluctuating free convection flow along heated horizontal circular cylinders". Int. J. Fluid Mech. Res., vol. 36, pp. 207-230, 2009.

[18] H. B. Keller, "Numerical methods in boundary layer theory", Аnnи. Rev. Fluid Mech., vol. 10, pp. 417-433, 1978.

[19] T. Cebeci, and P. Bradshaw, Physical and Computational Aspects of Convective Heat Transfer. Springer: Berlin Heidelberg, New York, 1984.

[20] P. Kumar, S. Rout, and P. S. Narayan, "Laminar natural convection boundary layers over bodies of arbitrary contour with vectored surface mass transfer", Int. J. Eng. Sci., vol. 10, pp. 1241-1252, 1989.

This is an open access article licensed under the terms of the Creative Commons Attribution Non-Commercial License (http://creativecommons.org/licenses/by-nc/3.0/) which permits unrestricted, non-commercial use, distribution and reproduction in any medium, provided the work is properly cited. 